\title{
Correction to: Spinor Fields, Singular Structures, Charge Conjugation, ELKO and Neutrino Masses
}

Luca Fabbri* (1)

Correction to: Adv. Appl. Clifford Algebras (2018) 28:7 https://doi.org/10.1007/s00006-018-0821-7

Unfortunately, the original version of this article was published with incorrect ORCID id for the author Luca Fabbri.

The correct ORCID id should be 0000-0002-9186-2807.

Luca Fabbri

DIME

Università di Genova

Genoa

Italy

e-mail: luca.fabbri@bo.infn.it

The original article can be found online at https://doi.org/10.1007/s00006-018-0821-7.

*Corresponding author. 